\title{
GÉNOTYPE GÉNÉRALISÉ ET SÉLECTION : ÉTUDE DE L'APPARIEMENT FRÈRE-SGEUR
}

C. ChEVALET. - Station centrale de Génétique animale, C.N.R.Z., 78-Jouy-en-Josas.

Les limites de l'étude de l'évolution d'une population d'effectif limité et soumise à l'influence de la sélection, tiennent dans l'insuffisance de la description de la population. Un progrès peut être

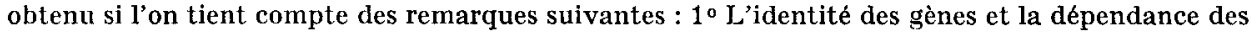
lois de probabilité qui leur sont attachées sont deux notions distinctes (Giblors M., 1966, Ann. Inst. Henri Poincaré, B, 2, 261-278); $2^{\circ} \mathrm{L}$ a sélection affecte les proportions relatives des génotypes et par là les relations de dépendance entre gènes. Une étude cohérente doit donc utiliser une description qui porte à la fois sur l'action des gènes et sur leur dépendance mutuelle. La notion de "génotype généralisé ", définie par GrLlois (1967, Ann. Génét., 10, 201-202) rend possible une telle description.

L'étude du cas particulier de la population formée à chaque génération d'un frère et d'une sœur, en croisement panmictique et sans mutation, a été abordée.à l'aide des notions précédentes. Cette étude fait appel à la technique des chaînes de Markov; elle a été conduite dans deux cas : $1^{\circ}$ absence de sélection; $2^{\circ}$ sélections gamétique et zygotique. Dans le premier cas, la chaine de MArkov est homogène, non ergodique; les résultats classiques sont retrouvés (extinction d'un gène, coefficients de parenté et de consanguinité égaux asymptotiquement à $1^{\circ}$ ). Dans le second cas, la chaîne de Markov est non homogène, non ergodique; les résultats de Malecot, dans le cas de la sélection faible, sont retrouvés $\left(1948\right.$, Les mathématiques de l'hérédité, Masson et $\left.\mathrm{C}^{1 \mathrm{e}}\right)$. Les notions utilisées permettent, en outre, de conduire les calculs sans approximation pour toutes intensités de sélection : on peut montrer, en particulier, que l'effet d'une sélection favorable à l'hétérozygote n'est sensible asymptotiquement, en l'absence de sélection gamétique, qu'au delà d'un certain taux, en deçà duquel l'effet de la consanguinité l'emporte.

\section{LA PANMIXIE DANS UNE TKACE DE CHEVAUX DOMESTIQUESS : L'EXEMPLE DES TROTTEURS FRANCAIS}

Ph. DREUX. - Laboratoire de Zoologie de l'École normale supérieure, 24, rue Lhomond, Paris-5

Les études génétiques quantitatives sur les animaux domestiques sont souvent difficiles et il peut être utile de les appuyer par des méthodes de génétique des populations. Pour cela, il est nécessaire de savoir si la race envisagée peut être assimilée à une population fermée panmictique. L'existence même d'un stud-book permet en général d'admettre qu'elle est à peu près fermée (Dreux, 1966, Ann. Génét., 9, 66).

Dans la race des trotteurs français, pour l'étude des caractères de la robe et des marques blanches, on a réuni 384 fratries issues de 768 reproducteurs. Pour tester la panmixie, ceux-ci ont été classés suivant les caractères qui nous intéressent $: 1^{\circ}$ la couleur de la robe : alezan $b b$ $(27,7 \%)$, bai $A B(50,2 \%)$, bai-brun à noir (génotypes probables : at $a^{t} B$, at $a B$, aaB ou rarement ED) $(22,1 \%$ ) (CASTI.E, 1954 , Genetics, 39,35$) ; 2^{\circ}$ la présence de marques $(88,7 \%)$ ou leur absence totale $(11,3 \%) ; 3^{\circ}$ la présence de marques en tête $(77,5 \%)$ ou leur absence $(22,5 \%) ; 4^{\circ}$ la présence d'au moins une balzane $(78,3 \%)$ ou l'absence totale $(21,7 \%)$.

Il est alors facile de calculer le nombre de croisements de chaque type (6 pour la couleur, 3 pour les autres caractères) que l'on obtiendrait s'ils n'étaient déterminés que par le hasard et de les comparer aux nombres de fratries correspondants observés effectivement. On obtient les nombres sujvants pour les 4 caractères étudiés : (1) 27-97-17-107-53-83 fratries observées 